\title{
Influence of Cardiac CT based disease severity and clinical symptoms on the diagnostic performance of myocardial perfusion
}

Nissen L MD^, Winther S MD, Ph.D*, Westra J BSc*, Ejlersen JA MD, Ph.D\#, Isaksen C MD ${ }^{\S}$, Rossi A MD, Ph.D ${ }^{\alpha}$, Holm NR MD*, Urbonaviciene G MD, Ph.D ${ }^{\dagger}$, Gormsen LC MD, Ph.D\%, Madsen LH MD, Ph.D^, Christiansen EH MD, Ph.D*, Maeng M MD, Ph.D*, Knudsen LL MD^, Frost L MD, Ph.D ${ }^{\dagger}$, Brix L MSc, Ph.D ${ }^{\S}$, Bøtker HE Professor*, Petersen SE MD DPHIL MPH ${ }^{£, \$}$ and Bøttcher M MD, Ph.D^

${ }^{\wedge}$ Department of Cardiology, Hospital Unit West Jutland, Herning, Denmark

* Department of Cardiology, Aarhus University Hospital, Aarhus, Denmark

\# Department of Nuclear Medicine, Hospital Unit West Jutland, Herning, Denmark

$\S$ Department of Radiology, Regional Hospital of Silkeborg, Silkeborg, Denmark

$\dagger$ Department of Cardiology, Regional Hospital of Silkeborg, Silkeborg, Denmark

\% Department of Nuclear Medicine, Aarhus University Hospital, Aarhus, Denmark

$£$ £illiam Harvey Research Institute, Queen Mary University of London, London, United Kingdom

\$ Barts Heart Centre, St. Bartholomew's Hospital, Barts Health NHS Trust, West Smithfield, London, United Kingdom

a Department of Biomedical Sciences - Humanitas University, Milan, Italy

\section{Institutions where the study was performed:}

Patients were enrolled at Department of Cardiology at Hospital Unit West Jutland, Herning Denmark and Department of Cardiology at Regional Hospital of Silkeborg, Silkeborg, Denmark. Invasive angiographies were performed at Department of Cardiology, Aarhus University Hospital, Aarhus, Denmark.

\section{Financial Support:}

This study was supported by the Danish Heart Foundation (grant no. 15-R99-A5837-22920) and the Health Research Fund of Central Denmark Region. SEP acknowledges support from the National Institute for Health Research Biomedical Research Centre at Barts.

\section{Disclosure:}

SEP received consultancy fees from Circle Cardiovascular Imaging Inc., Calgary, Alberta, Canada. 
NRH reports grants from Medis medical imaging during the conduct of the study; and grants and personal fees from Abbott, grants and personal fees from Boston Scientific, grants and nonfinancial support from Medis medical imaging in contexts other than the submitted work.

Word counts:

Abstract: 241

Word count: 3233 excl. tables and legends

\title{
Corresponding author:
}

Louise Nissen, MD

Department of Cardiology, Hospital Unit West, Herning, Denmark

G1. Landevej 61, 7400 Herning

Phone: +4526136430 , Fax: +4578452057 E-mail: lounisse@rm.dk

\begin{abstract}
Abbreviations
CCTA: Coronary Computed Tomographic Angiography, CACS: Coronary artery calcium score. CAD: Coronary Artery Disease, ICA: Invasive Coronary Angiography, CMR: Cardiac Magnetic Resonance, LGE: late gadolinium enhancement, MPS: Myocardial Perfusion Scintigraphy, FFR: Fractional Flow Reserve, PPV: Positive Predictive Value, QCA: Quantitative Coronary Analysis, ECG: Electrocardiography, SCCT: Society of Cardiovascular Computed Tomography, CACS: Coronary Artery Calcium Score, NPV: Negative Predictive Value, bpm: beats pr. minute, SSS: Summed Stress Score, SRS: Summed Rest Score, SDS: Summed Difference Score, CI: Confidence Interval, SPECT: Single-Photon Emission Computed Tomography, HR: Heart rate, OR: Odds ratio, PET: positron emission tomography, JOPCAB: J-incision off pump coronary artery bypass, PCI: percutaneous coronary intervention, CTO: coronary total occlusion, CABG: coronary artery bypass grafting, OMT: optimal medical therapy
\end{abstract}

\section{Authors' contributions}


LN, MB and SW participated in the conception and design of the study. CI obtained the CMR scans. LB provided assistance with the CMR scans and helped draft this manuscript. GU enrolled patients at Silkeborg Regional Hospital and analysed the CCTA scans. LF participated in the design and coordination of the study. JAE obtained the MPS scans. LHM and LLK enrolled patients and analysed CCTA scans at Hospital Unit West. NRH and JW coordinated the ICAs and collected data regarding this. EHC, MM and HEB conducted ICAs and FFR measurements at the Department of Cardiology, Aarhus University Hospital. SP and AR analysed the CMR scans as core lab. LCG analysed the MPS scans as core lab. All authors have critically reviewed, read and approved the final version of the manuscript.

\section{Acknowledgements}

The authors would like to thank Vibeke Lyngaard, Marianne Engbjerg Andersen, Stine Malling Søndergaard, Louise Jensen and Anne Sofie Mensberg (Dan-NICAD study nurses). The clinical staff at enrolling centres for their patience and collaboration. The BIAS Institute at Aarhus University kindly provided statistical assistance. SEP acknowledges support from the National Institute for Health Research Biomedical Research Centre at Barts. Finally, this study would not have been possible without the willing corporation of the patients in the Central Region of Denmark. 


\begin{abstract}
Objectives

We aimed to identify factors influencing the sensitivity of perfusion imaging after an initial positive coronary computed tomography angiography (CCTA) using invasive coronary angiography (ICA) with conditional fractional flow reserve (FFR) as reference. Secondly we aimed to identify factors associated with revascularisation and to evaluate treatment outcome after ICA.
\end{abstract}

\title{
Methods
}

We analysed 292 consecutive patients with suspected significant coronary artery disease (CAD) at CCTA, who underwent perfusion imaging with either cardiac magnetic resonance (CMR) or myocardial perfusion scintigraphy (MPS) followed by ICA with conditional FFR. Stratified analysis and uni- and multiple logistic regression analyses were performed to identify predictors of diagnostic agreement between perfusion scans and ICA and predictors of revascularisation.

\section{Results}

Myocardial ischemia evaluated with perfusion scans was present in 65/292 (22\%) while 117/292 (40\%) had obstructive CAD evaluated by ICA. Revascularisation rate was 90/292 (31\%). The overall sensitivity for perfusion scans was $39 \%$ (30-48), specificity $89 \%$ (83-93), PPV $69 \%$ (57$80)$ and NPV $68 \%$ (62-74). Stratified analysis showed higher sensitivities in patients with multivessel disease at CCTA $49 \%$ (37-60) and typical chest pain $50 \%$ (37-60). Predictors of revascularisation were multi-vessel disease by CCTA (OR 3.51 [1.91-6.48]) and a positive perfusion scan (OR 4.69 [2.49-8.83]).

\section{Conclusion}

The sensitivity for perfusion scans after CCTA was highest in patients with typical angina and multiple lesions at CCTA and predicted diagnostic agreement between perfusion scans and ICA. Abnormal perfusion and multi vessel disease at CCTA predicted revascularisation. 


\section{Introduction}

Despite guideline recommendations of non-invasive imaging testing for diagnosing stable coronary artery disease $(\mathrm{CAD})$ in patients with low/intermediate pre-test probability of CAD [1,2], invasive coronary angiography (ICA) continues to be used early on in the diagnostic pathway in patients with suspected CAD. Evidence from large populations presenting with chest pain has confirmed that the majority will not have obstructive CAD $[3,4]$. Moreover, a large US study reported that approximately $60 \%$ of elective ICAs found no obstructive CAD [5]. Avoiding unnecessary ICA would reduce patient risk and provide significant financial savings.

Non-invasive testing depicts anatomy, detects coronary atherosclerosis or identifies myocardial ischemia. Coronary computed tomography angiography (CCTA) assesses coronary anatomy [6] and is particularly useful for exclusion of CAD in low/intermediate-risk patients due to the high negative predictive value (NPV). Perfusion imaging e.g. cardiac magnetic resonance (CMR) or myocardial perfusion scintigraphy (MPS) assesses myocardial ischemia and the modalities provides strong prognostic information on mortality and morbidity $[7,8]$.

Several head-to-head studies have evaluated a combined diagnostic set-up with CCTA and perfusion imaging in high risk patients $[9,10]$.

In the Danish study of Non-Invasive testing in Coronary Artery Disease (Dan-NICAD) 1 trial $[11,12]$, the diagnostic accuracy of perfusion imaging with either CMR or MPS was tested in patients with significant CAD on CCTA, using ICA + conditional FFR measurements as the reference standard. In contrast to studies conducted in patients with high pre-test risk evaluated clinically, we found low sensitivity for both perfusion techniques [12].

The primary aim of this Dan-NICAD 1 sub-study was to evaluate if clinical parameters and CAD severity added independent predictive value to the diagnostic performance of perfusion imaging in patients with a low/intermediate risk of CAD and a CCTA suggestive of CAD. Secondarily, we evaluated the treatment outcome after ICA to further analyse the clinical consequence of potentially using perfusion imaging as a second line test instead of ICA. 


\section{Methods}

\section{Patients and study design}

This was a sub-study comprising all randomized patients from the Dan-NICAD 1 trial with a CCTA result suggesting obstructive CAD[12]. A detailed description of the Dan-NICAD 1 trial has been published [11]. In short, the trial was a two-centre, randomised, open label, head-to-head clinical trial including consecutive patients referred for CCTA with low/intermediate risk of CAD $(n=1675)$. Patients suspected of obstructive CAD at CCTA $(n=392)$ were randomized 1:1 to CMR or MPS and scheduled for subsequent ICA with conditional FFR. The core labs analysing the CMR and MPS scans were blinded to clinical information and the results of the ICA. Physicians performing ICA-FFR decided patient treatment blinded to the results of the perfusion scans. The study followed the Declaration of Helsinki. The Danish Data Protection Agency and the Central Denmark Regional Committee on Health Research Ethics approved the study. All patients provided informed written consent.

The study was registered at ClinicalTrials.gov (identifier: NCT02264717).

\section{Procedures}

\section{Coronary computed tomography angiography}

CCTA scans were performed using a 320 slice volume CT scanner (Aquillion One, Toshiba Medical Systems, Japan) with prospective electrocardiographic (ECG) gating. The images were analysed on a dedicated workstation (Vitrea Advanced Workstation, Vital Images, USA) by an experienced cardiologist. Luminal diameter stenosis was evaluated in each segment using the 18segment Society of Cardiovascular Computed Tomography (SCCT) model [13]. Coronary lesions were quantified for stenosis by visual assessment; and severity was classified as no stenosis: 0\% diameter reduction; mild stenosis: $1 \%$ to $29 \%$ diameter reduction; moderate stenosis: $30 \%$ to $49 \%$ diameter reduction; and severe stenosis: $50 \%$ to $100 \%$ diameter reduction. Severe stenosis and nonevaluable segments with a diameter exceeding $2 \mathrm{~mm}$ were defined as obstructive CAD.

\section{Myocardial perfusion scintigraphy}

MPS was obtained using a dedicated gamma camera (Cardio MD, Phillips Healthcare, Best, Holland). Patients conducted a standard symptom-limited bicycle exercise test preceded by 48 hours beta-blocker withdrawal. If patients were unable to reach target heart rate ( $\geq 85 \%$ of 220 -age), 
adenosine stress was performed. If stress images revealed any abnormalities, a resting study was obtained.

Images were analysed using Cedar Sinai AutoQuant software by an independent core lab (Department of Nuclear Medicine, Aarhus University Hospital, Aarhus, Denmark) using a 17segment model. For each segment, the perfusion defect was scored in a 5-point scoring system $(0$ : normal and up to 4: absence of tracer uptake) and summed into a summed stress score (SSS), summed rest score (SRS) and summed difference score (SDS). Images were also evaluated qualitatively with a binary outcome (normal/abnormal). An abnormal MPS scan was defined as 1) an SDS $\geq 4$ involving $\geq 2$ contiguous segments (reversible defect), 2) an SRS $\geq 4$ involving $\geq 2$ contiguous segments (irreversible defect) 3) a combination of 1) and 2) (mixed defect).

\section{Cardiac magnetic resonance imaging}

The CMR scans were conducted using a 1.5 Tesla system (Siemens MAGNETOM Avanto, Siemens Healthcare GmbH, Erlangen, Germany). Stress perfusion imaging was conducted after intravenous injection of either $0.4 \mathrm{mg}(5 \mathrm{ml})$ of Regadenosone ${ }^{\circledR}$ (Lexiscan, Astellas Pharma, USA) or intravenous adenosine-induced hyperaemia $140 \mu \mathrm{g} / \mathrm{kg} / \mathrm{min}$ over 4 minutes. A stepwise increase in adenosine dose was administered in case of inadequate response. For adenosine stress an increase of $>10 \mathrm{bpm}$ or a blood pressure reduction $>10 \mathrm{~mm} \mathrm{Hg}$ from baseline was considered sufficient [14]. For Regadenosone ${ }^{\circledR}$ an increase in heart rate of $>10 \mathrm{bpm}$ was considered a sufficient clinical response. Splenic switch-off was registered in all patients stressed with adenosine. As contrast agent, either Gadovist ${ }^{\circledR}$ (Bayer Schering Pharma AG, Germany) or Dotarem ${ }^{\circledR}$ (GD-DOTA, Guerbet LCC, USA), was used.

CMR analysis was performed in an independent core lab (William Harvey Research Institute, Queen Mary University of London, London, United Kingdom) blinded from additional patient information and results. Diagnostic confidence was evaluated using a scale from 1-3, indicating high, medium or low image quality. Images were evaluated qualitatively with a binary outcome (normal/abnormal) based on the perfusion analysis. Significant perfusion defects were defined as subendocardial or transmural signal changes by stress imaging or irreversible defects in $\geq 2$ contiguous segments by late gadolinium enhancement (LGE) imaging.

\section{Invasive coronary angiography with fractional flow reserve}

ICA was performed approximately 4 weeks after CCTA using a routine procedure. FFR measurements (St. Jude Medical, MN, USA and Volcano, San Diego, USA) were performed if 
technically possible in all coronary segments with a diameter $>2 \mathrm{~mm}$ where ICA showed a stenosis with a diameter $\geq 30$ and $<90 \%$ by visual assessment. All pressure waveforms were analysed by a core lab (Interventional Coronary Imaging Core Laboratory, Aarhus University Hospital, Denmark).

Two-dimensional (2D) quantitative coronary analysis (QCA) was performed in an independent core lab (ClinFact, Leiden, The Netherlands) using QAngioXA 7.3 QCA software. Haemodynamically obstructive CAD was defined as FFR $\leq 0.80$, or as a visual high-grade stenosis $(>90 \%$ diameter stenosis) in a vessel with a reference diameter above $\geq 2.0 \mathrm{~mm}$. If FFR was not technically possible, a QCA-measured stenosis exceeding $\geq 50 \%$ diameter stenosis defined a significant lesion.

\section{Statistical analysis}

Continuous variables were expressed using mean $( \pm \mathrm{SD})$ and categorical variables as frequency $(\mathrm{n}$, \%). Frequencies were compared by Fischer's exact test or Chi-square test as appropriate. The perfusion scans were analysed as one group due to the similar diagnostic performance of the CMR and MPS [12]. Only patients with complete data were included in the analyses. Data were binary grouped by sex, age (above/below 60 years), clinical presentation (+/- typical angina), CACS (above/below of 400) and single vs. multi-vessel disease by CCTA and the associated sensitivities, specificities and predictive values were calculated. These strata and the perfusion data were added to logistic regression models as independent variables to identify possible predictors of revascularisation after ICA (dependent variable). Model overfitting was balanced by limiting 1 variable in the multi-variable model for every 10 outcome. All results were presented with 95\% CI and two-sided p-value $<0.05$ were considered statistically significant.

Statistical analysis was performed using STATA-15 (StataCorp, College Station, TX, USA). 


\section{Results}

\section{Patient characteristics}

A total of 292 patients with suspected obstructive CAD on CCTA completed perfusion imaging with either CMR or MPS followed by ICA. Among these, 148 patients completed CMR and ICA and 144 completed MPS and ICA. Study flowchart and baseline characteristics are presented in Figure 1 and Table 1.

Imaging characteristics including CACS, CCTA, perfusion imaging and ICA are also presented in Table 1. Significant perfusion defects were identified in 65/292 (22\%) patients. In the 27 patients with a positive MPS, SSS, SRS and SDS were 11.2 $\pm 6.2,4.2 \pm 3.4$ and 7.6 \pm 5.3 , respectively (mean $\pm 2 \mathrm{SD}$ ). In the 38 patients with a positive CMR, the number of segments with a perfusion defect was $5.1 \pm 2.3$ (mean \pm 2 SD). The reference test (ICA with conditional FFR) classified 117/292 (40\%) patients with obstructive CAD, 75 due to a FFR $\leq 0.80$, the rest a high-grade stenosis. FFR was measured in 167 patients and mean FFR was $0.83 \pm 0.09$. Accuracy measures for the whole population are presented in Figure 2. Forty-five patients had coinciding abnormal perfusion imaging and obstructive CAD at ICA and 155 coinciding normal perfusion and non-obstructive CAD, Table 3 and Figure 2, yielding an accuracy of $68 \%$. Case examples of false negative and true positive CMR and MPS images are presented in Figure 3 and Figure 4.

Stratified analysis on patient related factors, coronary artery calcium score, and disease severity on the performance of perfusion imaging

Stratification was performed according to sex, age above or below 60 years, clinical presentation, CACS above or below 400 and single/multi vessel disease at CCTA as shown in Table 2. Except sensitivity and positive predictive value (PPV) for multi- vs. single vessel disease, the 95\% CI intervals overlapped (Marked with fat typing in table 2).

The proportion and absolute numbers of correctly classified patients with no CAD, one, two or three vessel disease is presented in Table 3. The proportion of correctly classified patients improved significantly for one vs. two vessel CAD $(p=0.025)$ and for one vs. three vessel CAD $(p=0.01)$.

Logistic regression models estimating predictors for diagnostic agreement between perfusion imaging and invasive coronary angiography and regression models predicting revascularisation 
The same variables used for stratification were also used in the regression models and the result of the perfusion imaging was included as a variable.

OR for diagnostic agreement between perfusion imaging and ICA, was significantly higher at multi-variable regression analysis for typical angina, $(\mathrm{p}=0.03)$ and multi-vessel disease by CCTA, $(\mathrm{p}<0.01)$. All results from uni- and multi-variable regression analysis are presented in Table 4. In the multi-variable regression analysis, significant predictors of revascularisation after ICA were multi-vessel disease by CCTA, $(p<0.01)$ and positive perfusion imaging, $(p<0.01)$. All results are presented in Table 5.

\section{Treatment consequence after invasive coronary angiography}

The clinical treatment consequences are presented in Figure 5. Of the 292 patients, 91 (31\%) were revascularized with percutaneous coronary intervention (PCI) $(47,52 \%)$, J-incision off pump coronary artery bypass (JOPCAB) $(7,8 \%)$, coronary total occlusion $(\mathrm{CTO})(4,4 \%)$ or coronary artery bypass grafting (CABG) $(33,36 \%)$. Another 27 patients $(9 \%)$ with abnormal ICA were treated medically based on a clinical decision, 5 of these had an abnormal perfusion scan.

Among the 91 revascularized patients, $40(44 \%)$ had a positive perfusion test and $51(56 \%)$ had a normal perfusion test. Revascularization was performed in $62 \%(40 / 65)$ patients with abnormal perfusion and in $22 \%(50 / 227)$ with a normal perfusion. Of the patients with normal perfusion, 32 underwent PCI, 4 JOPCAB (25 with one-vessel disease, 10 with two-vessel disease and 1 with three-vessel disease) and 14 CABG (7 with one-vessel disease, 5 with two-vessel disease and 2 with three-vessel disease). The revascularisation strategy was different as patients with abnormal perfusion underwent more extensive procedures (CTO and CABG) compared to patients with normal perfusion (PCI and JOPCAB), $\mathrm{p}=0.004$.

One patient undergoing PCI had a normal ICA according to our diagnostic criteria, but during the ICA a clinical decision of revascularisation was made 


\section{Discussion}

In the Dan-NICAD 1 trial, we found a sensitivity of $39 \%$ and a specificity of $89 \%$ for perfusion imaging (CMR and MPS) using ICA with conditional FFR as reference standard, in symptomatic patients with a CCTA suspected of obstructive CAD. Subgroup analyses in the present paper revealed that the sensitivity of perfusion imaging was slightly higher (around 50\%) in patients with typical angina and multi-vessel disease at CCTA. In accordance with this, we showed that typical angina and multi-vessel disease had an independent predictive value regarding abnormal ICA. Additionally, we noticed that multi-vessel disease at CCTA and a positive perfusion scan, but not typical angina, were independent predictors of revascularisation. Suggestively, only half of the revascularized patients, including patients undergoing $\mathrm{CABG}$, had abnormal perfusion.

Many former studies testing perfusion imaging with CMR or MPS against ICA with either QCA or FFR showed high sensitivities (70-90\%) and specificities (75-94 \%) [7,9,15-17]. The inclusion criteria and pre-test likelihood of CAD in these studies were diverse. In the present study, patients were included according to the CCTA findings, i.e. beforehand selected as suitable for this modality implying low/intermediate risk with a frequent prevalence of atypical symptoms and one-vessel disease (54\%) [12]. A recent Danish study including 143 patients from CCTA calculated similar diagnostic values of MPS including a sensitivity of $41 \%$, indicating that patients selected by CCTA have a lower ischemic burden than patients traditionally referred for MPS [18]. This is well understood considering that many high-risk patients are excluded from CCTA due to e.g. high BMI, high age, suspicion of high CACS (e.g. diabetics of whom we only have 22 (8\%) in the trial), irregular hear rhythm or kidney disease. Among the positive perfusion scans in the present study, 50/65 (77\%) covered 2-3 vessel territories, indicating that a larger ischemic burden is needed to cause substantial myocardial ischemia detected by perfusion imaging.

Obstructive multi-vessel disease by CCTA was an independent predictor of both diagnostic agreement between perfusion imaging and ICA and revascularisation. This is in concordance with the CONFIRM study showing multi-vessel disease to be the strongest predictor of ICA utilization after CCTA [19]. The results from the regression analysis indicate that disease severity and extent is part of the explanation for the low overall sensitivity in the Dan-NICAD trial, as most patients have one-vessel CAD and atypical symptoms. 
The Dan-NICAD 1 trial raises the question whether to manage patients according to the results of perfusion scan or ICA. Patient management can be driven by prognosis or symptoms. A strong argument for prognosis guided management is the widely accepted fact that a normal perfusion carries a favourable patient outcome [20-22]. A recent study evaluating a diagnostic strategy using positron emission tomography (PET) perfusion in patients with suspected CAD at CCTA [22] showed event rates 5 times higher for patients with an abnormal PET compared to a normal PET . In addition, event rates were similar for patients with a normal PET and an abnormal CCTA compared to patients with a normal CCTA. This study population resembles the Dan-NICAD patients and we expect a similar favourable outcome for patients with normal perfusion in our trial. Whether revascularisation with PCI improves patient prognosis in terms of mortality is debated. The COURAGE trial [23] evaluated the effect of revascularisation with PCI and optimal medical therapy (OMT) in patients with stable CAD or OMT alone and found no difference in terms of prognosis. Oppositely FAME 2 found reduced event rates in patients with stable CAD treated with FFR guided PCI and OMT compared to OMT alone [24]. The COURAGE trial nuclear sub-study showed that treatment with PCI + OMT in patients with myocardial ischemia at MPS reduced risk of death and cardiac events, especially in patients with moderate to severe ischemia [25]. Interestingly, if our patients had been treated according to the result of the perfusion scan, only $20 \%$ ((292-65)/292) would have been referred for ICA, presumably without impact of prognosis! Furthermore around half of the revascularisation procedures (50/90) would have been saved. These high percentages seem provocative and challenge current patient management strategies.

Performing symptom driven treatment, the anti-anginal effect of revascularisation is the primary goal. Little substantial knowledge exists regarding this management strategy, and the pathophysiology of angina is complex. It can be speculated that revascularisation of larger ischemic areas detected by MPS / CMR provides more pronounced symptom relief compared to treatment of smaller areas of ischemia although the supplying vessel to this area has a FFR below 0.8. However, we do not know whether the clinical benefit of revascularisation differed between patients with or without perfusion defects in our study population.

In our study 51 patients without a perfusion defect were revascularized; some will argued that these patients were missed calls by perfusion imaging and others will state that these patients were over treated. As FFR is measured in epicardial arteries $>2 \mathrm{~mm}$ and CMR/MPS detects ischemia at tissue level, it is possible that the discrepancy between the two examinations merely reflects the different nature of the modalities rather than the inferiority of the one over the over. 
Many years ago Diamond and Forrester published a highly cited paper on how to use probability as an aid in the clinical diagnosis of CAD[26]. Their data revealed that CAD was far more common among patients with typical angina compared to atypical angina and non-cardiac chest pain. Although questioning a patient about symptoms remains a corner stone in modern cardiology, we nowadays do not base diagnosis on clinical information alone. In this perspective, we find it interesting that out of other known high-risk markers of CAD (age, gender, CACS), typical angina turns out to be the one correlating with ischemia, emphasising that clinical history should not be forgotten in a world of advanced technology.

Given the discrepancy between the invasive and non-invasive techniques it seems that the population of patients with initially low to intermediate risk of CAD and a CCTA suggestive of obstructive CAD constitute a particular challenge for perfusion scans.

This study adds an important contribution to the on-going discussion on which diagnostic strategy to use in patients suspected of CAD and specifically address the problems evolving from comparing results of diagnostic methods evaluating CAD on a vessel level and myocardial level.

However, results from studies quantifying flow from perfusion imaging and estimating absolute flow during FFR will likely help to further clarify the discrepancy observed between the invasive and non-invasive techniques.

\section{Limitations}

Not all patients in this cohort obtained a complete dataset. Patients with incomplete data, approximately $25 \%$ of the randomized patients, did not complete the entire study protocol for various reasons specified in Figure 1. However this is not unexpected and similar findings were seen in e.g. the EVINCI study [27]. Patients did not drop out or were selected but the missing data mainly occurred due to logistic reasons as examinations were spread on multiple days and locations and this would not have influenced the prevalence of disease. Also FFR was not measured in all vessels but only in lesions with a diameter stenosis between (30-90\%) evaluated by the clinician performing the procedure. Performing FFR measures in all lesions might have changed the number of patients classified with CAD. Patients with a recent myocardial infarct, previous 
revascularisation with $\mathrm{PCI}$ or $\mathrm{CABG}$ were not included in this study and the results can therefore not be generalized to patients with known CAD.

\section{Conclusion}

In patients suspected of CAD and a CCTA with obstructive CAD, the sensitivity for perfusion scans were in general low compared to a reference standard of ICA with conditional FFR, but improved significantly in patients with typical chest pain symptoms and multi-vessel disease by CCTA. Multi-vessel disease at CCTA and abnormal perfusion predicted revascularisation. 
Table 1: Baseline characteristics and patient characteristics according to CACS, CCTA, perfusion imaging, and ICA

Patients per protocol

\begin{tabular}{|c|c|c|c|}
\hline & $\begin{array}{l}\text { Total } \\
(\mathrm{N}=\mathbf{2 9 2})\end{array}$ & $\begin{array}{l}\text { CMR } \\
(\mathrm{N}=148)\end{array}$ & $\begin{array}{l}\text { MPS } \\
(\mathrm{N}=144)\end{array}$ \\
\hline Gender, Women, $\mathrm{n}$ & $118(40 \%)$ & $60(41 \%)$ & $58(40 \%)$ \\
\hline Age, Y & $61.3 \pm 8.1$ & $61.9 \pm 7.9$ & $60.8 \pm 8.1$ \\
\hline BMI, mean & $26.9 \pm 3.9$ & $26.8 \pm 3.9$ & $27.1 \pm 4.0$ \\
\hline \multicolumn{4}{|l|}{ Risk factors } \\
\hline Current smoking & $52(18 \%)$ & $23(16 \%)$ & $29(20 \%)$ \\
\hline Antihypertensive treatment & $135(46 \%)$ & $75(51 \%)$ & $60(42 \%)$ \\
\hline Lipid-lowering treatment & $91(32 \%)$ & $48(33 \%)$ & $43(30 \%)$ \\
\hline Diabetes & $22(8 \%)$ & $14(10 \%)$ & $8(6 \%)$ \\
\hline Family history of early CAD\# & $123(42 \%)$ & $60(41 \%)$ & $63(44 \%)$ \\
\hline \multicolumn{4}{|l|}{ Symptoms } \\
\hline Typical angina & $94(32 \%)$ & $47(32 \%)$ & $47(33 \%)$ \\
\hline Atypical angina & $94(32 \%)$ & $52(35 \%)$ & $42(29 \%)$ \\
\hline Non-angina chest pain & $43(15 \%)$ & $20(14 \%)$ & $23(16 \%)$ \\
\hline Dyspnea or arrhythmia & $61(21 \%)$ & $29(20 \%)$ & $32(22 \%)$ \\
\hline \multicolumn{4}{|l|}{ Pretest probability* } \\
\hline Low $(<0.15)$ & $14(5 \%)$ & $8(5 \%)$ & $6(4 \%)$ \\
\hline Moderate $(>=0.15 \&<0.85)$ & $261(89 \%)$ & $132(89 \%)$ & $129(90 \%)$ \\
\hline $\operatorname{High}(<=0.85)$ & $17(6 \%)$ & $8(5 \%)$ & $9(6 \%)$ \\
\hline \multicolumn{4}{|l|}{ CACS } \\
\hline$-\mathrm{CACS}<400$ & $183(63 \%)$ & $88(60 \%)$ & $95(66 \%)$ \\
\hline - $\mathrm{CACS} \geq 400$ & $109(37 \%)$ & $60(40 \%)$ & $49(34 \%)$ \\
\hline \multicolumn{4}{|l|}{$C C T A^{*}$} \\
\hline - One-vessel CAD & $157(54 \%)$ & $77(52 \%)$ & $80(56 \%)$ \\
\hline - Two-vessel CAD & $75(26 \%)$ & $42(28 \%)$ & $33(23 \%)$ \\
\hline - Three-vessel or LM CAD & $59(20 \%)$ & $29(20 \%)$ & $30(21 \%)$ \\
\hline \multicolumn{4}{|l|}{ Perfusion imaging } \\
\hline - Abnormal perfusion & $65(22 \%)$ & $37(25 \%)$ & $27(19 \%)$ \\
\hline - Poor Image quality & $1(0.3 \%)$ & $1(0.7 \%)$ & $0(0 \%)$ \\
\hline - One-vessel territory & $14(5 \%)$ & $8(5 \%)$ & $6(4 \%)$ \\
\hline - Two-vessel territory & $37(13 \%)$ & $25(17 \%)$ & $12(8 \%)$ \\
\hline - Three-vessel territory & $13(4 \%)$ & $4(3 \%)$ & $9(7 \%)$ \\
\hline \multicolumn{4}{|l|}{$I C A$} \\
\hline CAD & $117(40 \%)$ & $59(40 \%)$ & $58(41 \%)$ \\
\hline - One-vessel CAD & $69(24 \%)$ & $33(22 \%)$ & $36(25 \%)$ \\
\hline - Two-vessel CAD & $32(11 \%)$ & $19(13 \%)$ & $13(9 \%)$ \\
\hline - Three-vessel or LM CAD & $16(5 \%)$ & $7(5 \%)$ & $9(6 \%)$ \\
\hline
\end{tabular}


Values are $\mathrm{n}(\%)$ or mean \pm SD. CMR: Cardiac magnetic resonance imaging, MPS: Myocardial perfusion scintigraphy BMI: body mass index, CCTA: Coronary computed tomography angiography. ICA: invasive coronary angiography.

CACS: coronary artery calcium score

\# Family history of coronary artery disease among 1'st degree relatives before the age of 60 .

* Pre-test probability assessed using the updated Diamond-Forrester score.

*One patient was randomized without a CCTA due to a high calciumscore. 
Table 2: Stratified analysis on diagnostic accuracy for perfusion imaging vs. ICA

\begin{tabular}{|c|c|c|c|c|c|}
\hline & Prevalence of CAD & Sensitivity & Specificity & PPV & NPV \\
\hline Female & $36 / 118(31 \%)$ & $33 \%(19-51)$ & $88 \%(79-94)$ & $55 \%(32-76)$ & $75 \%(65-83)$ \\
\hline Male & $81 / 174(47 \%)$ & $41 \%(30-52)$ & $89 \%(81-95)$ & $77 \%(61-88)$ & $63 \%(55-72)$ \\
\hline $\mathrm{AGE}<60$ & $45 / 110(41 \%)$ & $31 \%(18-47)$ & $85 \%(74-92)$ & $58 \%(37-78)$ & $64 \%(53-74)$ \\
\hline $\mathrm{AGE} \geq 60$ & $72 / 182(40 \%)$ & $43 \%(31-55)$ & $91 \%(84-96)$ & $76 \%(60-88)$ & $71 \%(63-78)$ \\
\hline $\begin{array}{l}\text { Typical } \\
\text { angina }\end{array}$ & $42 / 94(45 \%)$ & $50 \%(34-66)$ & $85 \%(72-93)$ & $72 \%(53-87)$ & $68 \%(55-79)$ \\
\hline Other* & $75 / 198(38 \%)$ & $32 \%(22-44)$ & $90 \%(84-95)$ & $67 \%(49-81)$ & $69 \%(61-76)$ \\
\hline $\mathrm{CACS}<400$ & $63 / 183(34 \%)$ & $32 \%(21-45)$ & $87 \%(79-92)$ & $56 \%(38-72)$ & $71 \%(63-78)$ \\
\hline $\mathrm{CACS} \geq 400$ & $54 / 109(50 \%)$ & $46 \%(33-60)$ & $93 \%(82-98)$ & $86 \%(68-96)$ & $64 \%(52-74)$ \\
\hline $\begin{array}{l}\text { Single-vessel } \\
\text { (CCTA) }\end{array}$ & $41 / 157(26 \%)$ & $20 \%(9-35)$ & $86 \%(79-92)$ & $33 \%(16-55)$ & $75 \%(67-82)$ \\
\hline $\begin{array}{l}\text { Multi-vessel } \\
\text { (CCTA) }\end{array}$ & $76 / 134(57 \%)$ & $49 \%(37-60)$ & $93 \%(83-98)$ & $90 \%(77-97)$ & $58 \%(47-68)$ \\
\hline
\end{tabular}

Stratified analyses on sex, age \pm 60 , typical angina symptoms or *other covering atypical, non-specific, dyspnoea or arrhythmia, $\mathrm{CACS} \pm 400$ and single vs. multi-vessel disease classified by CCTA.

Values are proportions and $95 \% \mathrm{CI}$ are shown in the parentheses. All analyses are per-patient.

CAD: coronary artery disease, PPV: positive predictive value, NPV: negative predictive value, CACS: coronary artery calcium score, CCTA: coronary computed tomography tomography. 
Table 3: Patients with single or multi-vessel CAD by ICA

\begin{tabular}{|l|lccc|}
\cline { 2 - 5 } & \multicolumn{4}{|c|}{ Perfusion imaging } \\
\cline { 2 - 5 } & Normal & Abnormal & Total \\
\cline { 2 - 5 } & No CAD & $155(89 \%)$ & 20 & 175 \\
One-vessel CAD & 50 & $19(28 \%)$ & 69 \\
Two-vessel CAD & 16 & $16(50 \%)$ & 32 \\
Three-vessel CAD or & 6 & $10(63 \%)$ & 16 \\
LM CAD & 227 & 65 & 292 \\
Total & & & \\
\cline { 2 - 5 }
\end{tabular}

Numbers in parentheses are proportions of correctly classified patients. The total proportion of correctly classified patients are $(155+19+16+10) / 29268 \%$.

CAD: coronary artery disease, ICA: invasive coronary angiography LM: Left main 
Table 4: Simple and multiple logistic regressions to identify predictors of diagnostic agreement between perfusion imaging and ICA

\begin{tabular}{lcc}
\hline \multicolumn{1}{|c}{ Variable (Reference group) } & $\begin{array}{c}\text { Simple logistic regression } \\
\text { model, } \mathbf{O R}\end{array}$ & $\begin{array}{c}\text { Multiple logistic regression } \\
\text { model, OR }\end{array}$ \\
\hline Female & $1.40(0.60-3.13 ; \mathrm{p}=0.45)$ & $1.61(0.65-3.95 ; \mathrm{p}=0.30)$ \\
Male & $1.67(0.76-3.67 ; \mathrm{p}=0.19)$ & $1.34(0.55-3.25 ; \mathrm{p}=0.52)$ \\
\hline Age $<\mathbf{6 0}$ & & \\
Age $\geq \mathbf{6 0}$ & $2.13(0.98-4.61 ; \mathrm{p}=0.06)$ & $2.57(1.11-5.98 ; \mathrm{p}=0.03)$ \\
\hline Chest pain other* & & \\
Typical & $1.85(0.87-3.94 ; \mathrm{p}=0.12)$ & $0.95(0.38-2.36 ; \mathrm{p}=0.91)$ \\
\hline CACS $<\mathbf{4 0 0}$ & & \\
CACS $\geq \mathbf{4 0 0}$ & & $4.53(1.61-12.71 ; \mathrm{p}<0.001)$ \\
\hline Single-vessel by CCTA & $3.91(1.60-9.57 ; \mathrm{p}<0.001)$ & \\
\hline Multi-vessel by CCTA &
\end{tabular}

Simple and multiple logistic regression models to identify predictors of diagnostic agreement between perfusion imaging and ICA. Values are (95\% CI; p-value)

*Other covering atypical, non-specific, dyspnoea or arrhythmia

ICA: Invasive coronary angiography, OR: odds ratio, CACS: coronary artery calcium score, CCTA: coronary computed tomography angiography. 
Table 5: Simple and Multiple logistic regression predictors of revascularisation

\begin{tabular}{lll}
\hline Variable (Reference group) & $\begin{array}{c}\text { Simple logistic regression } \\
\text { model OR }\end{array}$ & $\begin{array}{c}\text { Multiple logistic regression } \\
\text { model OR }\end{array}$ \\
\hline Female & $1.70(1.01-2.86 ; \mathrm{p}=0.05)$ & $1.47(0.82-2.64 ; \mathrm{p}=0.19)$ \\
Male & $0.89(0.54-1.48 ; \mathrm{p}=0.65)$ & $0.67(0.37-1.21 ; \mathrm{p}=0.19)$ \\
$\begin{array}{l}\text { Age }<\mathbf{6 0} \\
\text { Age } \geq \mathbf{6 0}\end{array}$ & $1.31(0.77-2.20 ; \mathrm{p}=0.32)$ & $1.06(0.58-1.94 ; \mathrm{p}=0.84)$ \\
$\begin{array}{l}\text { Chest pain other* } \\
\text { Typical }\end{array}$ & $2.24(1.35-3.72 ; \mathrm{p}=0.002)$ & $1.47(0.80-2.72 ; \mathrm{p}=0.21)$ \\
\hline CACS $<\mathbf{4 0 0}$ & & $3.51(1.91-6.48 ; \mathrm{p}<0.001)$ \\
CACS $\geq \mathbf{4 0 0}$ & & \\
$\begin{array}{l}\text { CCTA CAD extent (Single- } \\
\text { vessel) }\end{array}$ & $4.40(2.58-7.52 ; \mathrm{p}<0.001)$ & $4.69(2.49-8.83 ; \mathrm{p}<0.001)$ \\
\hline $\begin{array}{l}\text { Multi-vessel } \\
\text { No perfusion defect }\end{array}$ & & \\
Perfusion defect & $5.52(3.06-9.95 ; \mathrm{p}<0.001)$ & \\
\hline
\end{tabular}

Simple and multiple logistic regression models to identify predictors of revascularisation. Values are (95\% CI; p-value) *Other covering atypical, non-specific, dyspnoea or arrhythmia

ICA: Invasive coronary angiography, OR: odds ratio, CACS: coronary artery calcium score, CCTA: coronary computed tomography angiography. 
Figure 1: Patient flowchart.

CCTA: coronary computed tomography angiography, CMR: cardiac magnetic resonance, MPS: myocardial perfusion scintigraphy, ICA: invasive coronary angiography, CACS: coronary artery calcium score.

Figure 2: Results of perfusion imaging compared with invasive angiography with conditional FFR

2.a Sensitivity, specificity, PPV and NPV measured at the patient level for the perfusion imaging. Results are presented with $95 \%$ CI.

2.b: Results in absolute numbers at patient level.

PPV: positive predictive value, NPV: negative predictive value, ICA: invasive coronary angiography.

Figure 3: Two case examples of CCTA, MPS and ICA findings

Upper example; a false negative MPS: CCTA showed diffuse mixed calcified and soft plaques throughout LAD. Both rest and stress MPS were normal without perfusion defects. ICA showed an elongated severe stenosis in LAD visually assessed as $>90 \%$ stenosis. Patient was treated with a graft, LIMA to LAD.

Lower example; a true positive MPS: CCTA showed a severe proximal stenosis in LAD, the plaque was mixed soft and calcified. Stress and rest MPS showed both fixed and reversible perfusion defects in the anterior wall. ICA showed an occluded LAD with only retrograde contrast filling. The patient was treated with PCI in the proximal LAD.

CCTA: coronary computed tomography angiography. MPS: myocardial perfusion scintigraphy. ICA: invasive coronary angiography. LAD: left anterior descending artery. LIMA: left internal mammary artery. PCI: percutaneous coronary intervention.

Figure 4: Two case examples of CCTA, CMR and ICA findings

Upper example; a false negative CMR: CCTA showed diffuse mixed calcified and soft plaques throughout LAD. Stress CMR was normal without hypo perfused areas. ICA showed an elongated stenosis in LAD, FFR was 0.72 . The patient was treated with a graft, LIMA to LAD

Lower example; a true positive CMR; CCTA showed severe calcified plaques throughout LAD. Stress CMR showed a large transmural perfusion defect in the antero-septal wall. ICA showed an occluded LAD with only retrograde contrast filling. The patient was treated with $\mathrm{CABG}$, grafts for $\mathrm{LAD}$ and RCA.

CCTA: coronary computed tomography angiography. CMR: cardiac magnetic resonance, ICA: invasive coronary angiography. LAD: Left anterior descending artery. FFR: fractional flow reserve. LIMA: left internal mammary artery. CABG: coronary artery bypass grafting. RCA: right coronary artery.

Figure 5: Treatment consequence of ICA stratified by the results of perfusion imaging ICA: invasive coronary angiography, JOPCAB: J-incision off pump coronary artery bypass, PCI: percutaneous coronary intervention, CTO: coronary total occlusion, CABG: coronary artery bypass grafting. 


\section{References}

[1] Moss AJ, Williams MC, Newby DE, Nicol ED. The Updated NICE Guidelines: Cardiac CT as the First-Line Test for Coronary Artery Disease. Curr Cardiovasc Imaging Rep. 2017;10(5):15,0179412-6. Epub 2017 Mar 27; doi:10.1007/s12410-017-9412-6 [doi].

[2] Task Force Members, Montalescot G, Sechtem U, Achenbach S, Andreotti F, Arden C, et al. 2013 ESC guidelines on the management of stable coronary artery disease: the Task Force on the management of stable coronary artery disease of the European Society of Cardiology. Eur Heart J. 2013;34(38):2949-3003; doi:10.1093/eurheartj/eht296 [doi].

[3] Hannan EL, Samadashvili Z, Cozzens K, Walford G, Holmes DR,Jr, Jacobs AK, et al. Appropriateness of diagnostic catheterization for suspected coronary artery disease in New York State. Circ Cardiovasc Interv. 2014;7(1):19-27; doi:10.1161/CIRCINTERVENTIONS.113.000741 [doi].

[4] Patel MR, Dai D, Hernandez AF, Douglas PS, Messenger J, Garratt KN, et al. Prevalence and predictors of nonobstructive coronary artery disease identified with coronary angiography in contemporary clinical practice. Am Heart J. 2014;167(6):846,52.e2; doi:10.1016/j.ahj.2014.03.001 [doi].

[5] Patel MR, Peterson ED, Dai D, Brennan JM, Redberg RF, Anderson HV, et al. Low diagnostic yield of elective coronary angiography. N Engl J Med. 2010;362(10):886-95;

doi:10.1056/NEJMoa0907272 [doi].

[6] Bettencourt N, Nagel E. Comparison of MR and CT for the Assessment of the Significance of Coronary Artery Disease: a Review. Current Cardiovascular Imaging Reports. 2013;6(2):102-16; doi:10.1007/s12410-012-9186-9.

[7] Underwood SR, Anagnostopoulos C, Cerqueira M, Ell PJ, Flint EJ, Harbinson M, et al. Myocardial perfusion scintigraphy: the evidence. Eur J Nucl Med Mol Imaging. 2004;31(2):261-91; doi:10.1007/s00259-003-1344-5 [doi]. 
[8] Morton G, Schuster A, Perera D, Nagel E. Cardiac magnetic resonance imaging to guide complex revascularization in stable coronary artery disease. Eur Heart J. 2010;31(18):2209-15; doi:10.1093/eurheartj/ehq256 [doi].

[9] Groothuis JG, Beek AM, Brinckman SL, Meijerink MR, van den Oever ML, Hofman MB, et al. Combined non-invasive functional and anatomical diagnostic work-up in clinical practice: the magnetic resonance and computed tomography in suspected coronary artery disease (MARCC) study. Eur Heart J. 2013;34(26):1990-8; doi:10.1093/eurheartj/eht077 [doi].

[10] Ponte M, Bettencourt N, Pereira E, Ferreira ND, Chiribiri A, Schuster A, et al. Anatomical versus functional assessment of coronary artery disease: direct comparison of computed tomography coronary angiography and magnetic resonance myocardial perfusion imaging in patients with intermediate pre-test probability. Int J Cardiovasc Imaging. 2014;30(8):1589-97; doi:10.1007/s10554-014-0492-y [doi].

[11] Nissen L, Winther S, Isaksen C, Ejlersen JA, Brix L, Urbonaviciene G, Frost L, Madsen LH, Knudsen LL, Schmidt SE, Holm NR, Maeng M, Nyegaard M, Bøtker HE, Bøttcher M.

Danish study of Non-Invasive testing in Coronary Artery Disease (DanNICAD): study protocol for arandomised controlled trial.. Trials. 2016 May 26;17(1):262. doi: 10.1186/s13063-016-1388-z. 2016;17(262).

[12] Nissen L, Winther S, Westra J, Ejlersen JA, Isaksen C, Rossi A, et al. Diagnosing coronary artery disease after a positive coronary computed tomography angiography: the Dan-NICAD open label, parallel, head to head, randomized controlled diagnostic accuracy trial of cardiovascular magnetic resonance and myocardial perfusion scintigraphy. Eur Heart J Cardiovasc Imaging. 2018;19(4):369-77; doi:10.1093/ehjci/jex342 [doi].

[13] Raff GL, Abidov A, Achenbach S, Berman DS, Boxt LM, Budoff MJ, et al. SCCT guidelines for the interpretation and reporting of coronary computed tomographic angiography. J Cardiovasc Comput Tomogr. 2009;3(2):122-36; doi:10.1016/j.jcct.2009.01.001 [doi].

[14] Karamitsos TD, Ntusi NAB, Francis JM, Holloway CJ, Myerson SG, Neubauer S. Feasibility and safety of high-dose adenosine perfusion cardiovascular magnetic resonance. J Cardiovasc Magn Reson. 2010;12(1):66; doi:10.1186/1532-429X-12-66.

[15] Greenwood JP, Maredia N, Younger JF, Brown JM, Nixon J, Everett CC, et al. Cardiovascular magnetic resonance and single-photon emission computed tomography for diagnosis of coronary heart disease (CE-MARC): a prospective trial. Lancet. 2012;379(9814):453-60; doi:10.1016/S01406736(11)61335-4 [doi].

[16] Li M, Zhou T, Yang LF, Peng ZH, Ding J, Sun G. Diagnostic accuracy of myocardial magnetic resonance perfusion to diagnose ischemic stenosis with fractional flow reserve as reference: systematic review and meta-analysis. JACC Cardiovasc Imaging. 2014;7(11):1098-105; doi:10.1016/j.jcmg.2014.07.011 [doi]. 
[17] Danad I, Szymonifka J, Twisk JWR, Norgaard BL, Zarins CK, Knaapen P, et al. Diagnostic performance of cardiac imaging methods to diagnose ischaemia-causing coronary artery disease when directly compared with fractional flow reserve as a reference standard: a meta-analysis. Eur Heart J. 2017;38(13):991-8; doi:10.1093/eurheartj/ehw095 [doi].

[18] Sand NPR, Veien KT, Nielsen SS, Norgaard BL, Larsen P, Johansen A, et al. Prospective Comparison of FFR Derived From Coronary CT Angiography With SPECT Perfusion Imaging in Stable Coronary Artery Disease: The ReASSESS Study. JACC Cardiovasc Imaging. 2018;11(11):1640-50; doi:S1936-878X(18)30437-6 [pii].

[19] Shaw LJ, Hausleiter J, Achenbach S, Al-Mallah M, Berman DS, Budoff MJ, et al. Coronary Computed Tomographic Angiography as a Gatekeeper to Invasive Diagnostic and Surgical Procedures: Results From the Multicenter CONFIRM (Coronary CT Angiography Evaluation for Clinical Outcomes: An International Multicenter) Registry. Journal of the American College of Cardiology. 2012;60:2103-14.

[20] Greenwood JP, Ripley DP, Berry C, McCann GP, Plein S, Bucciarelli-Ducci C, et al. Effect of Care Guided by Cardiovascular Magnetic Resonance, Myocardial Perfusion Scintigraphy, or NICE Guidelines on Subsequent Unnecessary Angiography Rates: The CE-MARC 2 Randomized Clinical Trial. JAMA. 2016;316(10):1051-60; doi:10.1001/jama.2016.12680 [doi].

[21] Douglas PS, Hoffmann U, Patel MR, Mark DB, Al-Khalidi HR, Cavanaugh B, et al. Outcomes of Anatomical versus Functional Testing for Coronary Artery Disease. N Engl J Med. 2015; doi:10.1056/NEJMoa1415516 [doi].

[22] Maaniitty T, Stenstrom I, Bax JJ, Uusitalo V, Ukkonen H, Kajander S, et al. Prognostic Value of Coronary CT Angiography With Selective PET Perfusion Imaging in Coronary Artery Disease. JACC Cardiovasc Imaging. 2017;10(11):1361-70; doi:S1936-878X(17)30260-7 [pii].

[23] Sedlis SP, Hartigan PM, Teo KK, Maron DJ, Spertus JA, Mancini GBJ, et al. Effect of PCI on Long-Term Survival in Patients with Stable Ischemic Heart Disease. N Engl J Med. 2015;373(20):1937-46; doi:10.1056/NEJMoa1505532.

[24] De Bruyne B, Pijls NH, Kalesan B, Barbato E, Tonino PA, Piroth Z, et al. Fractional flow reserve-guided PCI versus medical therapy in stable coronary disease. N Engl J Med. 2012;367(11):991-1001; doi:10.1056/NEJMoa1205361 [doi].

[25] Shaw LJ, Berman DS, Maron DJ, Mancini GB, Hayes SW, Hartigan PM, et al. Optimal medical therapy with or without percutaneous coronary intervention to reduce ischemic burden: results from the Clinical Outcomes Utilizing Revascularization and Aggressive Drug Evaluation (COURAGE) trial nuclear substudy. Circulation. 2008;117(10):1283-91; doi:10.1161/CIRCULATIONAHA.107.743963 [doi]. 
[26] Diamond GA. Analysis of probability as an aid in the clinical diagnosis of coronary-artery disease. N Engl J Med. 1979;300(24):1350-8.

[27] Liga R, Vontobel J, Rovai D, Marinelli M, Caselli C, Pietila M, et al. Multicentre multi-device hybrid imaging study of coronary artery disease: results from the EValuation of INtegrated Cardiac Imaging for the Detection and Characterization of Ischaemic Heart Disease (EVINCI) hybrid imaging population. Eur Heart J Cardiovasc Imaging. 2016;17(9):951-60; doi:10.1093/ehjci/jew038 [doi]. 
Figure 1

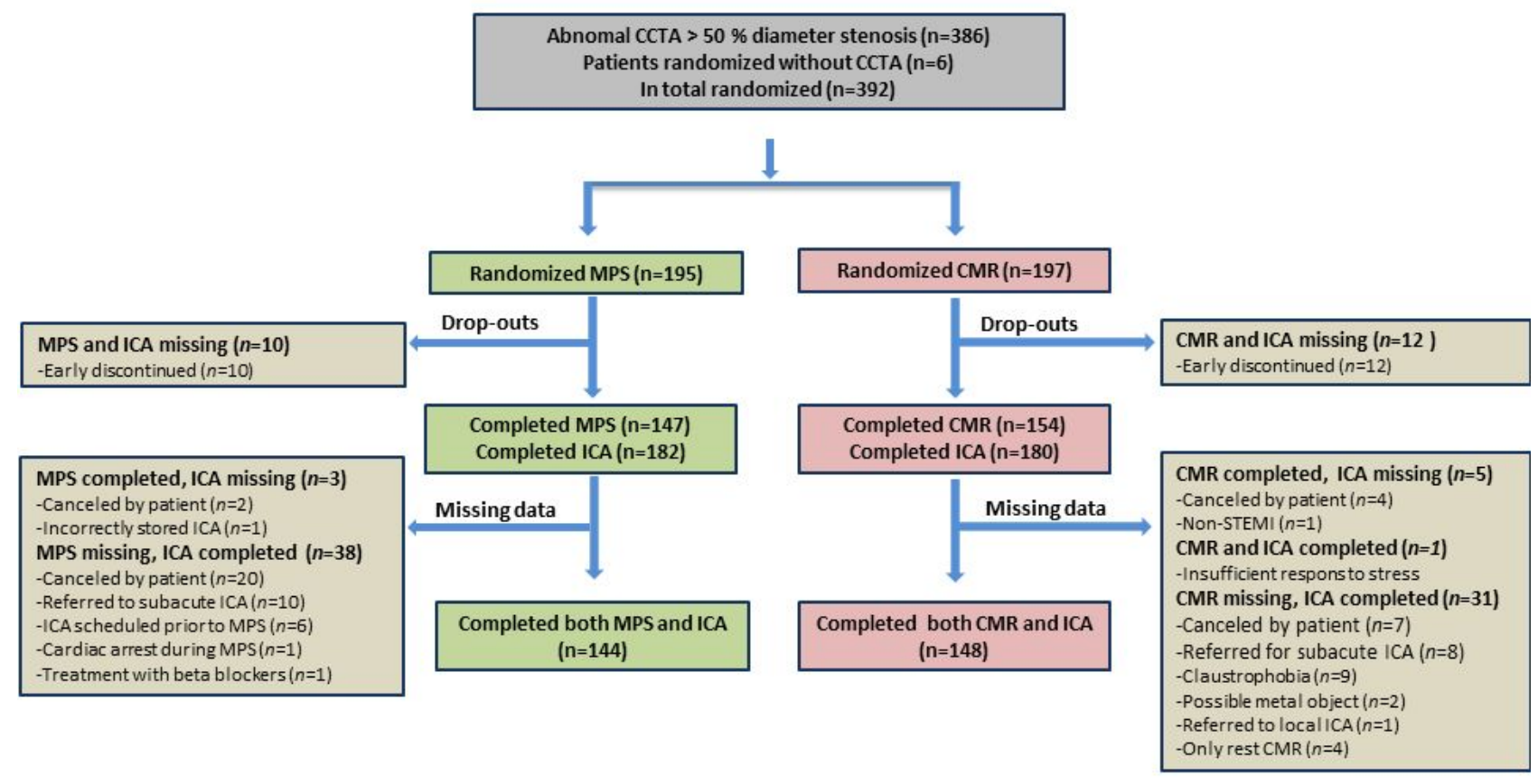




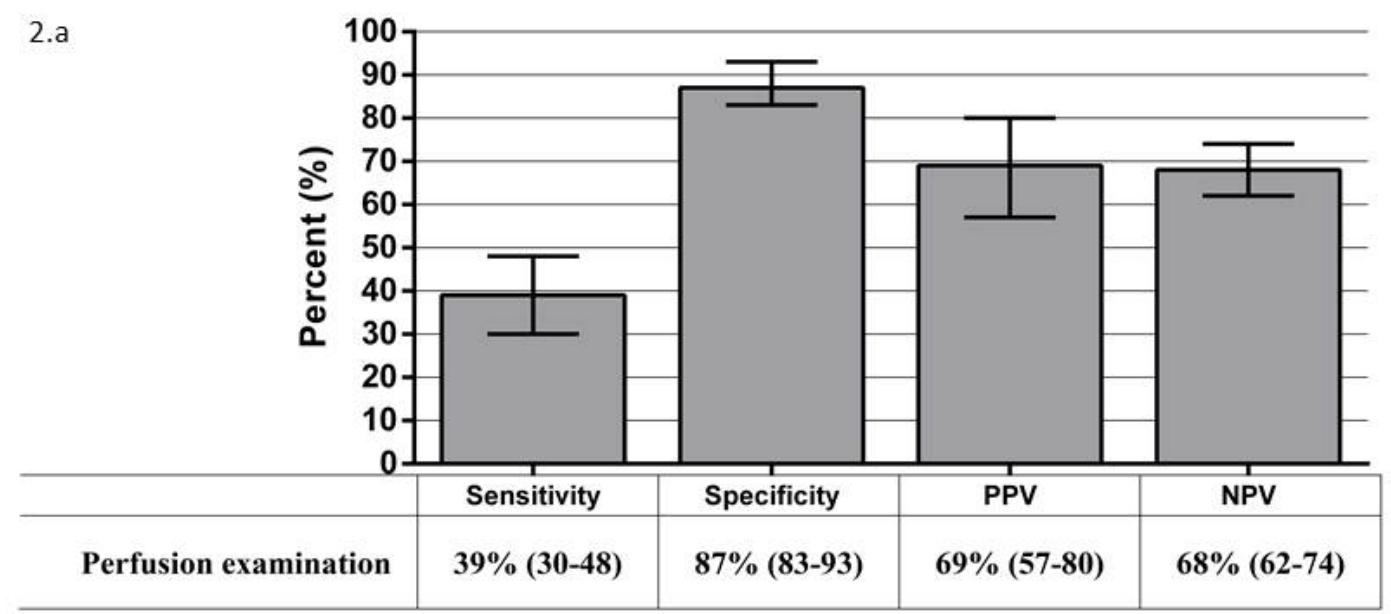




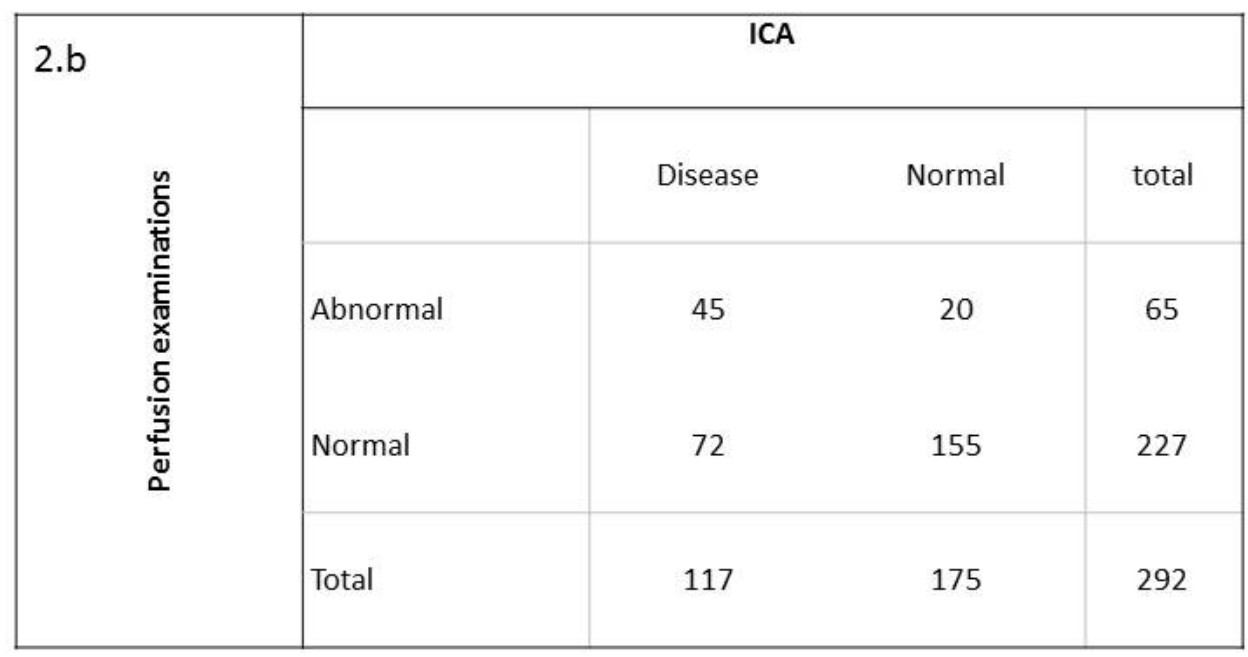


Figure 3

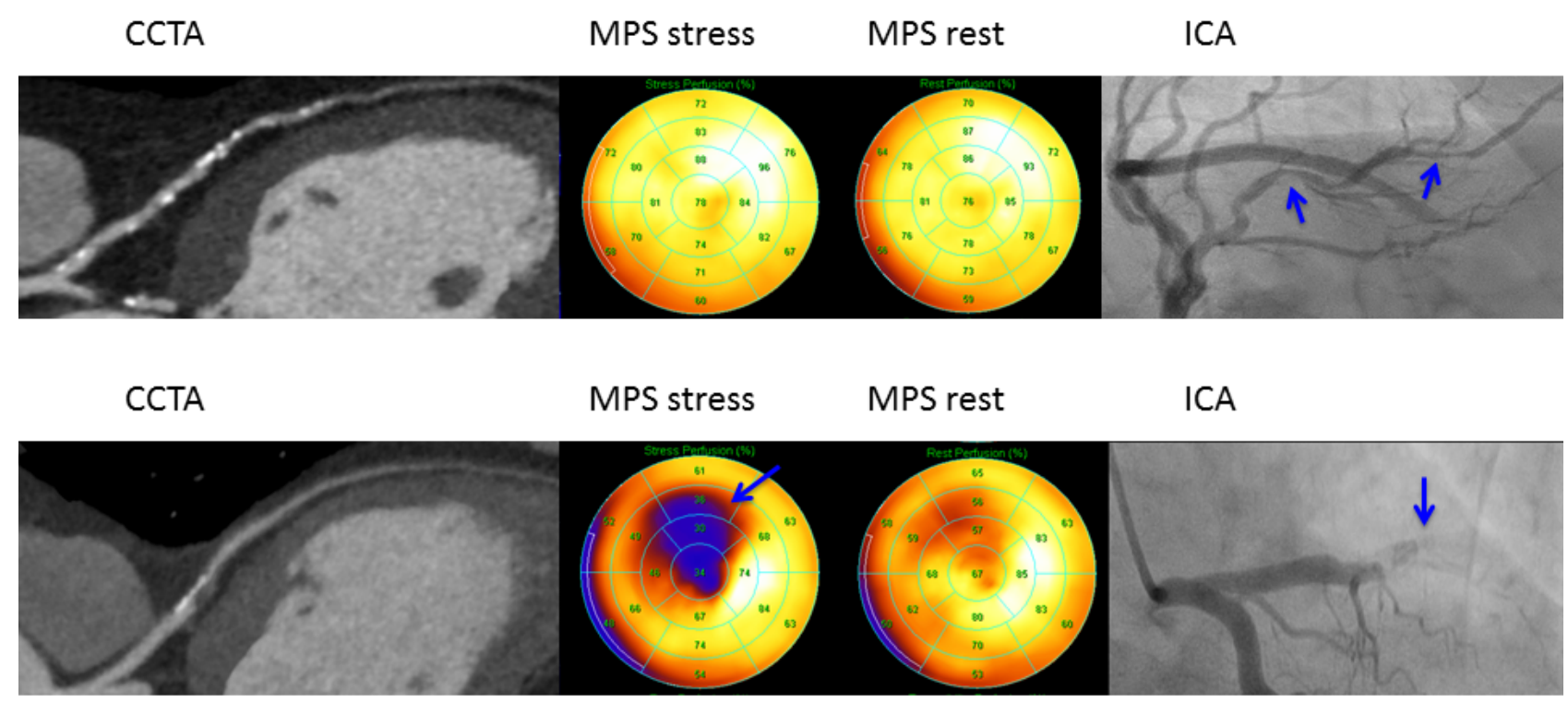


Figure 4

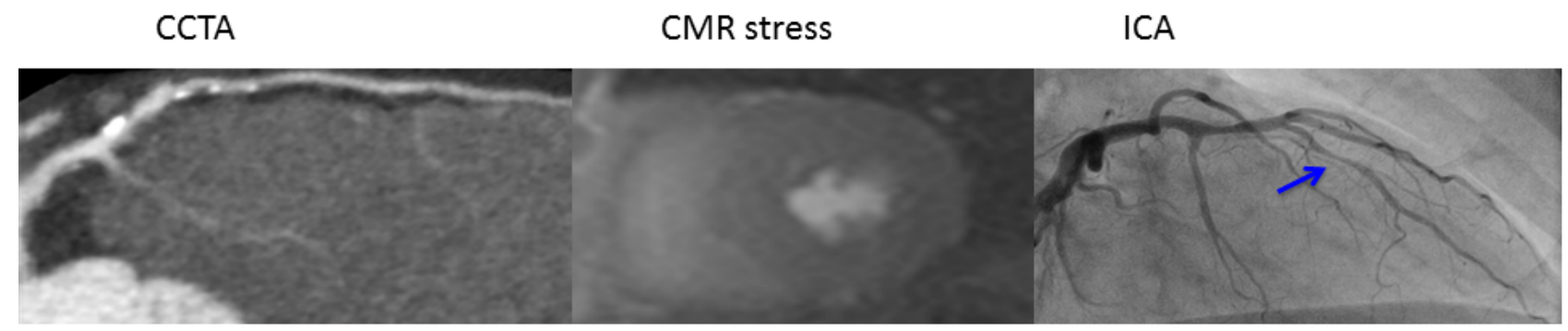

CCTA

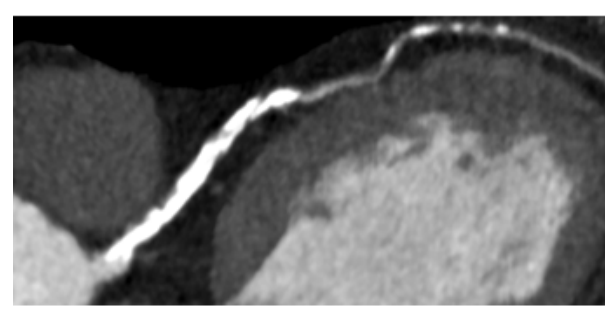

CMR stress

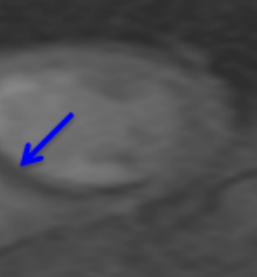

ICA

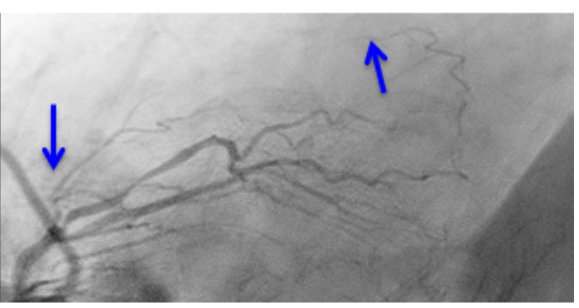


Figure 3

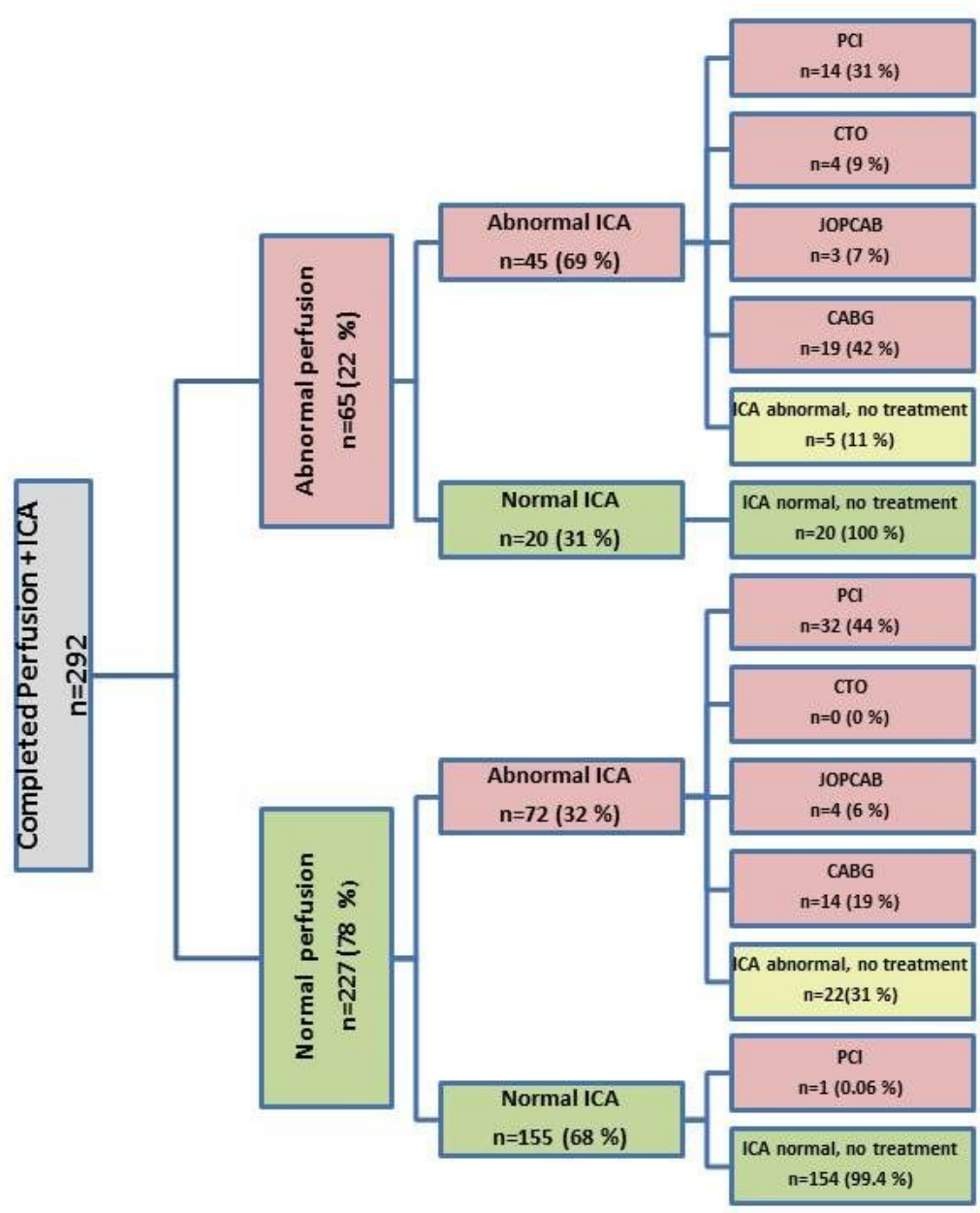

